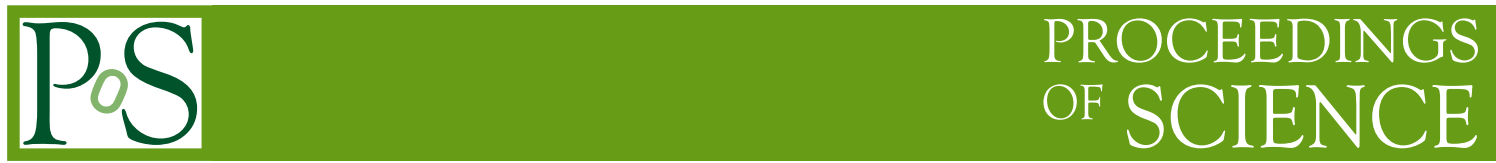

\title{
Alignment of the ATLAS Inner Detector tracking system
}

\author{
Louise SKINNARI* \\ On behalf of the ATLAS Collaboration \\ University of California, Berkeley \\ E-mail: louise.skinnariecern.ch
}

\begin{abstract}
To reconstruct trajectories of charged particles produced in the LHC collisions, the ATLAS detector is equipped with an inner tracking system built using two different technologies, silicon planar sensors (pixels and microstrips) and drift-tube based detectors. Together they constitute the ATLAS Inner Detector, which is embedded in a $2 \mathrm{~T}$ solenoidal field. An alignment of the inner tracking system, accurately determining the position and orientations of individual detector modules, is necessary for achieving required performance. The ATLAS Inner Detector has been aligned using isolated, high- $p_{T}$ collision tracks, and using cosmic-ray tracks collected between LHC proton-proton collisions. These proceedings present the alignment procedure, its results, and performance with LHC collision data during 2011. Results from real data are compared with Monte Carlo simulation of a perfectly aligned detector.
\end{abstract}

XXIst International Europhysics Conference on High Energy Physics

21-27 July 2011

Grenoble, Rhône-Alpes France

\footnotetext{
*Speaker.
} 


\section{Introduction}

The ATLAS detector [1] is equipped with an inner tracking system which consists of three independent, yet complimentary, subsystems: the Pixel detector, the Semiconductor Tracker (SCT), and the Transition Radiation Tracker (TRT). Built using different techniques (silicon pixels, silicon microstrips, and drift tubes), these constitute the ATLAS Inner Detector (ID).

Accurate vertex finding, reconstructing trajectories of charged particles, and precisely measuring track momenta is of crucial importance for physics analyses. To achieve its scientific goals, an alignment of the inner tracking system is required to determine accurately almost 36,000 degrees of freedom for the silicon detectors and 700,000 for the TRT. The baseline goal of the ID alignment is to determine the position and orientation of detector modules with high precision such that the limited knowledge of sensor locations should not deteriorate the resolution of track parameters by more than $20 \%$ with respect to the intrinsic tracker resolution. Precision measurements require even higher accuracy of a few microns.

\section{Alignment procedure}

The alignment is performed in three stages as (1) relative alignment of subdetectors, (2) alignment of separate layers in a subdetector, and (3) alignment of individual modules or straws within each detector layer [2]. To minimize multiple scattering effects, the alignment relies on high- $p_{T}$ tracks from collision and cosmic-ray events. Collision tracks with $p_{T}>9 \mathrm{GeV}$ are selected online by the High-Level Trigger and written to a special calibration stream.

The alignment is based on track-hit residuals, the distance between the extrapolated track position on a given module to the recorded hit position in the same module. Two independent alignment algorithms have been developed. Global $\chi^{2}$ alignment involves a simultaneous fit of all track and alignment parameters by minimizing a $\chi^{2}$, resulting in a linear system with a size of the number of alignment degrees of freedom. In local $\chi^{2}$ alignment, instead each detector module is aligned separately, requiring the solution of a system of up to 6 linear equations for each module.

Various constraints may be imposed during the alignment including beamspot and vertex constraints and knowledge from survey assembly data, such as pixel module deformations. A powerful, recently employed method is to derive momentum constraints based on the ratio of energy to momentum $(E / p)$ for high- $p_{T}$ electrons. Constraining the ID momentum measurement based on information from the Muon Spectrometer is an additional potential method.

\section{Global systematic distortions}

Some global systematic detector distortions leave track-hit residuals unaltered for tracks originating from the interaction region. Standard track-based alignment algorithms are insensitive to such misalignments. Several approaches for removing systematic biases may be considered, including different track topologies where tracks from cosmic-ray or beam-halo events are used, or tracks collected during times when the solenoidal field was off. Another approach is to apply additional constraints during the alignment procedure, such as constraining tracks to the beamspot or a common vertex, using the momentum as measured by the Muon Spectrometer, $E / p$ constraints, or physics based constraints from the invariant mass of resonance decays. 
The $Z$ resonance, with its decay to oppositely charged muons, provides a clean signature and a powerful tool for studying alignment performance and probing systematic effects. With wellknown intrinsic resolution, the estimated resolution of the dimuon invariant mass is a measure of detector effects. Muons from $Z$ decays tend to have considerably higher $p_{T}$ and are less sensitive to systematic effects in the material description compared to lower mass resonances. $Z$ candidate events are selected for these studies using isolated muons with $p_{T}>20 \mathrm{GeV}$. The invariant mass is formed from ID track parameters to probe alignment effects in the inner tracking system.

For the summer 2011 data reprocessing, constraints derived from electron $E / p$ were imposed during the alignment procedure. The goal was to reduce large observed momentum biases, particularly in the endcap regions. Preliminary results indicate significantly improved momentum systematics, shown in Figure 1.
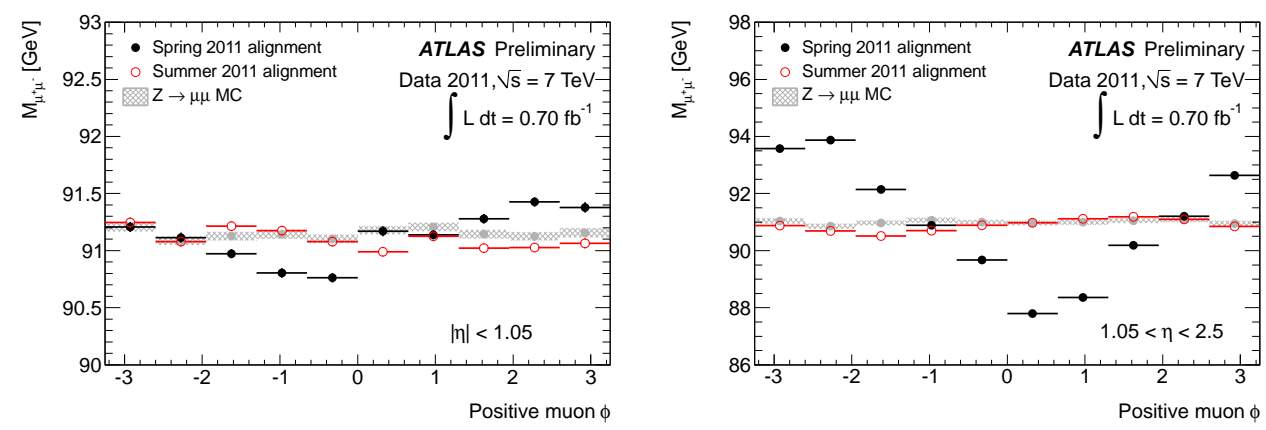

Figure 1: Mean $Z$ invariant mass versus $\phi$ for positive muons, where both muons have $|\eta|<1.05$ (left) or $1.05<\eta<2.5$ (right). Filled black points show data processed with spring 2011 alignment, open red circles data processed with summer 2011 alignment, and gray dashed areas are ideal alignment performance based on Monte Carlo simulations [3].

\section{Outlook}

The ATLAS Inner Detector has been aligned using well isolated, high- $p_{T}$ tracks from $7 \mathrm{TeV}$ LHC collision data, complemented by cosmic-ray tracks collected with cosmic triggers between the LHC collisions. The ID alignment performance is approaching design resolutions and focus is now shifting towards understanding more subtle effects such as global systematic detector distortions. Clear improvements in alignment performance are observed after performing the alignment using constraints from electron $E / p$.

\section{References}

[1] The ATLAS Collaboration, The ATLAS Experiment at the CERN Large Hadron Collider, JINST 3 S08003 (2008).

[2] The ATLAS Collaboration, Alignment of the ATLAS Inner Detector Tracking System with 2010 LHC proton-proton collisions at $\sqrt{s}=7 \mathrm{TeV}$, ATLAS-CONF-2011-012 (2011), http://cdsweb.cern.ch/record/1334582.

[3] Inner tracking combined performance plots of the ATLAS Collaboration, https://twiki.cern.ch/twiki/bin/view/AtlasPublic/InDetTrackingPerformanceApprovedPlots. 